\title{
Reliable e-nose for air toxicity monitoring by filter diagonalization method
}

\author{
Ricardo Macías-Quijas ${ }^{1}$, Ramiro Velázquez ${ }^{1}$, Roberto de Fazio $^{2}$, Paolo Visconti ${ }^{2}$, \\ Nicola Ivan Giannoccaro ${ }^{2}$, Aimé Lay-Ekuakille ${ }^{2}$ \\ ${ }^{1}$ Facultad de Ingeniería, Universidad Panamericana, Aguascalientes, Mexico \\ ${ }^{2}$ Department of Innovation Engineering, University of Salento, Lecce, Italy
}

\begin{tabular}{l} 
Article Info \\
\hline Article history: \\
Received Jul 9, 2021 \\
Revised Sep 14, 2021 \\
Accepted Oct 10, 2021 \\
\hline Keywords: \\
Electronic nose (e-nose) \\
Filter diagonalization method \\
MOS gas sensor \\
Sensor characterization \\
Toxic compounds
\end{tabular}

\begin{abstract}
This paper introduces a compact, affordable electronic nose (e-nose) device devoted to detect the presence of toxic compounds that could affect human health, such as carbon monoxide, combustible gas, hydrogen, methane, and smoke, among others. Such artificial olfaction device consists of an array of six metal oxide semiconductor (MOS) sensors and a computer-based information system for signal acquisition, processing, and visualization. This study further proposes the use of the filter diagonalization method (FDM) to extract the spectral contents of the signals obtained from the sensors. Preliminary results show that the prototype is functional and that the FDM approach is suitable for a later classification stage. Example deployment scenarios of the proposed e-nose include indoor facilities (buildings and warehouses), compromised air quality places (mines and sanitary landfills), public transportation, mobile robots, and wireless sensor networks.
\end{abstract}

This is an open access article under the $\underline{C C B Y-S A}$ license.

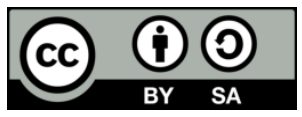

\section{Corresponding Author:}

Ramiro Velázquez

Facultad de Ingeniería, Universidad Panamericana

Josemaría Escrivá de Balaguer 101, Aguascalientes, 20290, Mexico

Email: rvelazquez@up.edu.mx

\section{INTRODUCTION}

An electronic nose (e-nose) is a sensing instrument comprising a set of different gas sensors that react when exposed to a wide range of chemical particles. According to the outputs obtained from the sensors, many conclusions about air quality and toxicity can be obtained. They have shown great promise and utility in three domains: food control, disease diagnosis, and environmental monitoring. In food control, enoses have been devoted toward assuring quality and safety for consumers. Some features already addressed in this domain are food freshness, ageing, contamination during processing, shelf life, and authenticity confirmation. Viejo et al. [1] implemented an e-nose to assess the aroma profiles in beer and automate the industrial quality inspection process. Similarly, Radi et al. [2] developed an e-nose for classifying odors from synthetic flavors such as grapes, strawberry, mango, and orange. To alert about rancidity, Xu et al. [3] introduced an e-nose monitoring the changes of pecans during storage. Timsorn and Wongchoosuk [4] explored the use of an e-nose device to identify odors from formalin contamination in seafood. To prevent products' adulteration, Świgło and Chmielewski [5] proposed an e-nose to assist in the authenticity testing of products such as meat, honey, milk, and plant oils. To discourage meat dealers from committing food fraud, Laga and Sarno [6] presented an e-nose discriminating pork from beef. Wang et al. [7] deployed an e-nose inside a domestic refrigerator to assess the food freshness level of fruits, vegetables, and meat.

The ability of humans to detect diseases with smell has played a significant role in clinical diagnosis. E-noses can ease the detection of volatile organic compounds (VOC) exhibiting bacterial 
pathogens and have the potential to become a valuable disease diagnosis tool for humans, animals, and plants. Sanchez et al. [8] monitored exhaled human breath with an e-nose to help diagnose and monitor certain digestive and respiratory diseases. Siyang et al. [9] analyzed the odor of human urine samples with an e-nose to detect diseases such as diabetes. In response to the current COVID-19 pandemic, airbus is deploying e-nose devices in its aircrafts to detect the virus [10]. In veterinary medicine, Jia et al. [11] explored an e-nose device for detecting three types of wound infections in rats. To detect bovis-infected cattle in farms, Peled et al. [12] reported the use of an e-nose to analyze VOC in breath samples. In botanics, Baietto et al. [13] explored the use of an e-nose to sense the bole-rot fungi that affects trees. Wilson [14] developed and tested an e-nose for the rapid identification of insecticide residues in crops.

Nowadays, e-noses in environmental monitoring have found application in four fields: i) air quality, ii) water quality, iii) process control, and iv) odor control systems [15]. Some representative work include Wongchoosuk's WiFi e-nose sensing and quantifying indoor air contaminants even in very low concentrations [16]. The e-nose prototype proposed by Mishra devoted to identify poison gases emanated from waste [17]. The work of Baby reporting on the use of an e-nose to sense contaminating residues and pesticides in water [18]. The system in [19] using different gas sensors, monitors gas concentration and temperature in a biogas reactor. The smart system proposed by [20] was capable of sensing ammonium nitrate that could lead to fire and explosion in storage warehouses. Applications of e-noses outside these three main domains also involve explosive detection [21] and the space industry [22].

Within the context of air quality assessment, this paper presents the characterization and implementation of a novel, affordable e-nose device. The prototype consists of a set of six metal oxide semiconductor (MOS) gas sensors capable of detecting, i) combustible gas, ii) alcohol, iii) methane, iv) carbon monoxide, v) hydrogen, and vi) smoke, which might represent a threat for the human health. The implemented device has small dimensions allowing for seamless integration into mobile robots, indoor facilities, urban transport, risky environments (such as mines and sanitary landfills), or sensor networks monitoring broad surface areas.

Similar e-nose prototypes exploring MOS sensors can be found in the literature [1], [16], [23]-[25]; sensor arrays range from four to ten, depending on the application. Prototypes rely on MOS technology because it offers small-size and robust sensors, quite good sensitivity, simple signal processing, commercial availability, and low cost. The main difference across devices is the method for data processing. Approaches such as artificial neural networks (ANN), principal component analysis (PCA), Fourier transform, and wavelets have been explored with satisfactory results for further developing predictive models.

In this paper, the sensors' signals were spectrally analyzed using the filter diagonalization method (FDM). To our knowledge, this work is the first one that reports on the use of FDM for e-nose signal processing. The remainder of this work paper is structured as follows: section 2 introduces the e-nose device, the MOS sensors used, the main system components, the FDM, and its implementation in the prototype. In section 3, the experiments that have been carried out are described and the results are shown. Finally, the concluding remarks and the future work perspectives are given in section 4 .

\section{RESEARCH METHOD}

An operational prototype was implemented with low-cost materials, and it is the first approach for the research work. The proposed device involves six gas sensors, each one focusing on a specific gas. They capture the components present in the air for later off-line analysis. The device comprises an electronic module for data acquisition and a software to visualize the sensors' behavior. To ensure an adequate gas concentration around the e-nose, two items have also been considered: an air pump conveying the samples and a hermetic box enclosing the device.

\subsection{Experimental apparatus}

A 3D printed plastic base was designed to host the set of sensors. It is small $(6.5 \times 5 \times 7 \mathrm{~cm})$ and displays six perforations around its side-faces for the proper installation of the MOS sensors as shown in Figure 1(a). All electronic boards and electrical connections are placed inside the plastic base allowing a clean and safe device handling as shown in Figure 1(b).

The prototype employs six different types of MOS sensors (Hanwei Electronics Co., Ltd., Henan, China), which change their electric resistance when its sensing material comes in contact with the gas. Figure 2 illustrates the MOS sensors external and internal structures. The external structure as shown in Figure 2(a) involves a mesh-like enclosure that protects the sensing material and filters out the suspended particles on the air, so that only gaseous elements access into the chamber. The clamping ring secures the mesh and serves as the base for the sensing material and the electrical connections.

Internally, the sensor is composed of a tin dioxide $\left(\mathrm{SnO}_{2}\right)$ layer, which is the sensing material that reacts to the input gas. In clean air, no electric current flows through this layer, but when gas is detected, 
electrons are released, allowing current to flow. The current output signal is conveyed via platinum $(\mathrm{Pt})$ wires as shown in Figure 2(b). Using a simple voltage-divider configuration, the gas concentrations can be derived. Each of the six MOS sensors comprised in the prototype is sensitive to a specific gas providing valuable data upon the analysis of their responses. We previously reported a complete experimental characterization of the MOS sensors [26]. Characteristics such as sensitivity, behavior to temperature, and step response were examined. Figure 3 presents, as an example, the behavior obtained from the alcohol (ethanol) gas sensor (S2). Figure 3(a) shows its resistance ratio Rs/Ro; here, Rs represents the sensor resistance to the target gas $\left(\mathrm{C}_{2} \mathrm{H}_{5} \mathrm{OH}\right)$ given a specific concentration, while Ro represents the sensor resistance in clean ambient air. Note that the resistance ratio Rs/Ro decreases as the concentration of the gas increases. Figure 3(b) shows how the sensitivity curve of the sensor is affected by temperature. Here, Rso represents the resistance of the sensor in $125 \mathrm{ppm}$ (parts-per-million) alcohol at $20^{\circ} \mathrm{C}$. Note that the resistance ratio Rs/Rso decreases as environmental temperature increases. Figure 3(c) shows the sensor response to a step input of $125 \mathrm{ppm}$ alcohol gas. Note that $\mathrm{S} 2$ delivers $3.5 \mathrm{~V}$ in the presence of the gas and $0.5 \mathrm{~V}$ (the baseline) in the absence of gas.

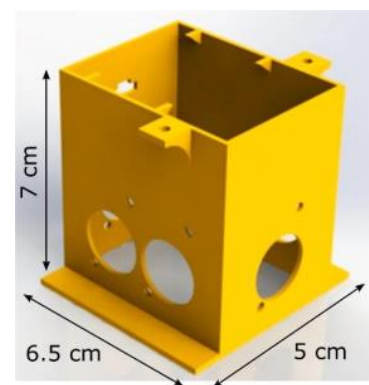

(a)

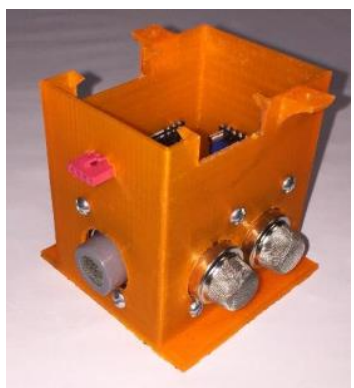

(b)

Figure 1. The plastic base structure: (a) 3D design and (b) actual prototype

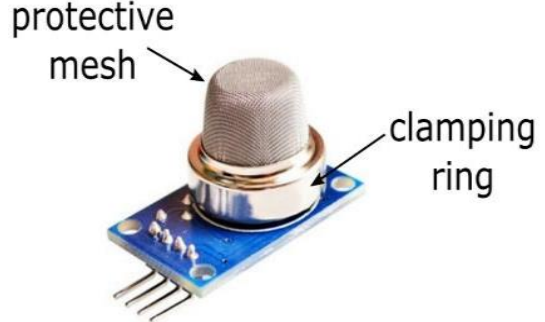

(a)

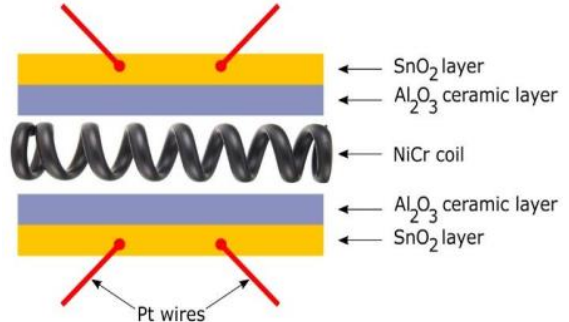

(b)

Figure 2. MOS sensors: (a) external and (b) internal structures

Table 1 lists the MOS sensors used, their model number, and their target detection gases. Note that the sensors are sensitive to a primary gas but also react to secondary gases, resulting in an overlap of their responses as shown in Figure 3(a) and Table 1. This overlap is advantageous since it provides more information about the chemical compounds contained in the air sample, thus facilitating the signal classification stage.

The prototype also comprises an electronic module which encompasses a 32-bit microcontroller (Texas instruments Stellaris LM4F120) responsible for the sensors' signal acquisition, universal serial bus (USB) interfacing with a computer, and the external air pump control. Figure 4(a) shows the block-level diagram of the electronic module while Figure 4(b) shows the electrical diagram for the air pump control. To synchronize the start and end of signal collection and transfer the acquired signals for processing and visualization, a simple communication protocol was built between the electronic module and the computer.

Figure 5 shows the activity diagram of the system operation. The computer initiates the process which lasts $180 \mathrm{~s}$. During this time interval, the sensor array collects data from its vicinity. At $\mathrm{t}=20 \mathrm{~s}$, the pump starts introducing air into the hermetic box. At $t=50 \mathrm{~s}$, the pump is set off; the sensors reset (i.e., signals start going to their baseline). The electronic module captures this behavior as well. 
Figure 6 illustrates all six sensors' responses detailing the different stages. Note that the response of S2 (alcohol gas sensor) is the slowest one both in its rising and recovery times. This result is due to the low volatility that alcohol exhibits compared to the other substances explored.

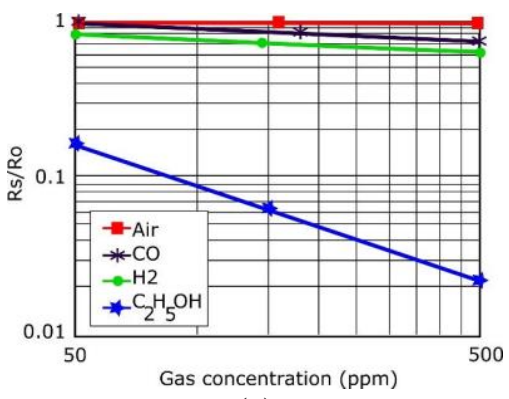

(a)

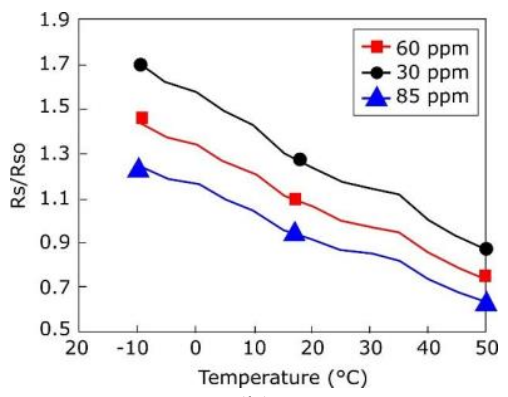

(b)

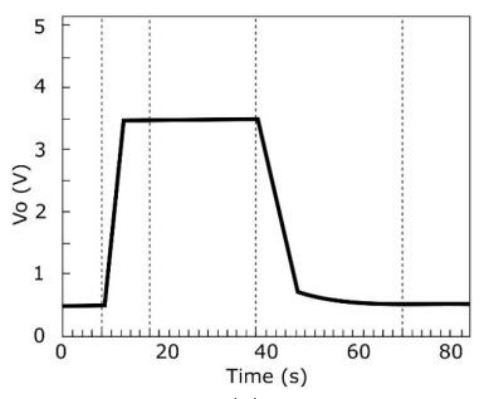

(c)

Figure 3. Sensor characterization example for S2 (alcohol sensor), (a) sensitivity curve, (b) behaviour to temperature, and (c) step response

Table 1. Specifications of the MOS sensors in the e-nose

\begin{tabular}{cccc}
\hline Sensor & Hanwei Item & Primary Gas & Secondary Gases \\
\hline S1 & MQ2 & Combustible gas & $\mathrm{H}_{2}, \mathrm{LPG}, \mathrm{CH}_{4}, \mathrm{CO}$, Alcohol, Propane \\
S2 & MQ3 & Alcohol & $\mathrm{CO}, \mathrm{H}_{2}$ \\
S3 & MQ4 & Methane & Propane, Butane, Alcohol \\
S4 & MQ7 & Carbon monoxide & $\mathrm{H}_{2}, \mathrm{LPG}, \mathrm{CH}_{4}$ \\
S5 & MQ8 & Hydrogen & $\mathrm{CO}$ \\
S6 & MQ135 & Air quality & $\mathrm{NH}_{3}, \mathrm{NOx}$, Alcohol, Benzene, Smoke, $\mathrm{CO}_{2}$ \\
\hline
\end{tabular}

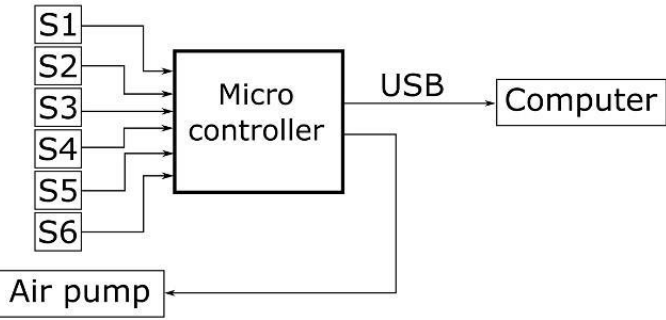

(a)

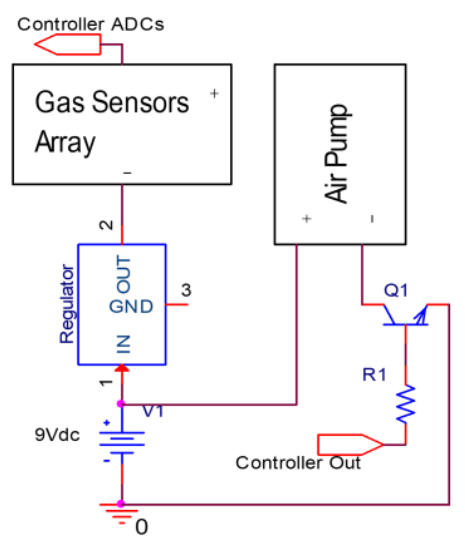

(b)

Figure 4. E-nose system: (a) block-level diagram and (b) electrical diagram of the air pump control

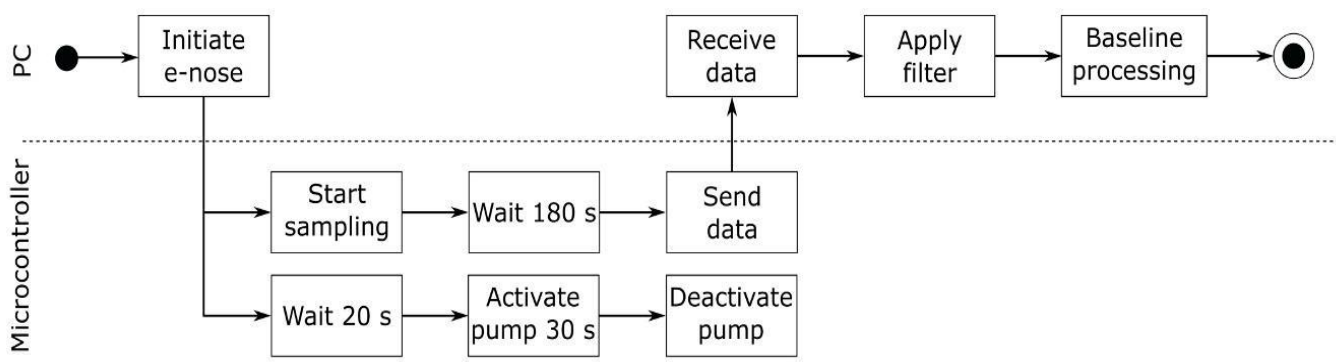

Figure 5. The activity diagram for the e-nose software 


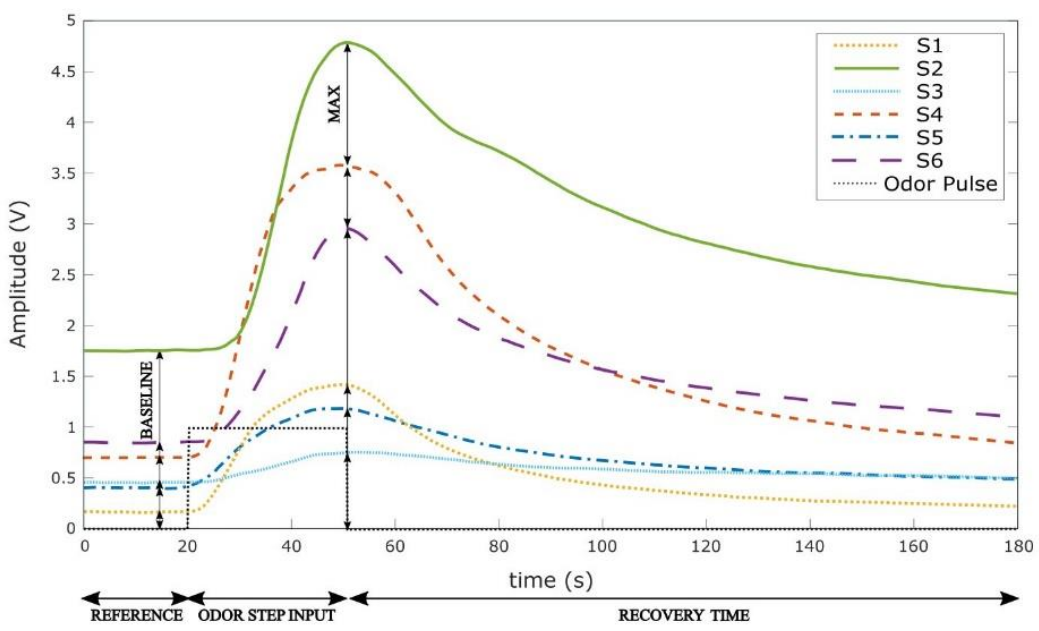

Figure 6. The sensors' response during the acquisition process

Once the $180 \mathrm{~s}$ acquisition process has concluded, the microcontroller gathers the sensors readings, converts the continuous data into digital form, and builds a data frame which is sent to the computer via USB. The computer further filters the samples with a ten-tap moving average filter to eliminate the undesirable peaks in the sensors' responses. To obtain reliable plots with comparable maximum and minimum values, the sensors' individual baselines were determined and eliminated using (1):

$$
y_{i}=y_{i}-y_{i 0}
$$

where $y_{i}$ represents the output of the $\mathrm{i}^{\text {th }}$-sensor and $y_{i 0}$ its baseline.

The final prototype is shown in Figure 7(a). Note that the microcontroller is fitted in the plastic base. The necessary connections between the sensor array and the electronic module are located inside. Figure 7(b) shows the implemented system. Inside the hermetic box, the e-nose (plastic base, sensor array, and electronic module) can be seen. Outside it, the air pump conveying the air samples can be appreciated. The air pump control circuitry and the power supply are also shown. The final prototype exhibits compact dimensions as shown in Figure 1(a); low mass (500 g), and low cost (150 USD).

\subsection{FDM analysis}

The filter diagonalization method (FDM) was initially developed for quantum dynamics calculations [27] and later used for nuclear magnetic resonance (NMR) signal processing [28], and leak detection in pipelines [29], [30]. It provides a nonlinear parametric method for time-domain signal analysis using a sum of damped sinusoids. FDM is traditionally used to solve harmonic inversion problems (HIP), delivering highresolution spectra. Compared to Fourier analysis, it is not limited by the incertitude principle, thus providing high-quality spectra together with a high signal to noise ratio (SNR) without needing a large number of samples.

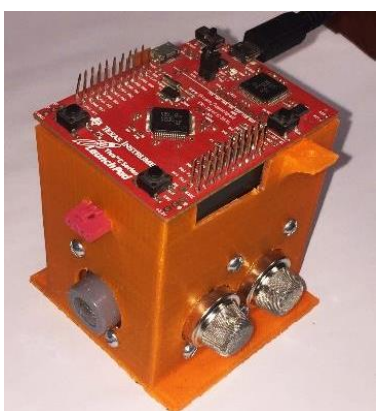

(a)

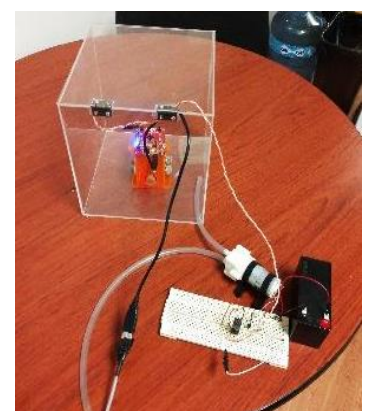

(b)

Figure 7. First prototype is (a) control unit and sensors in the plastic base and (b) the prototype with the external air pump and the hermetic box 


\subsubsection{FDM formulation}

To explain the method, let us consider a complex unidimensional signal $c_{n}=c(n \tau)$ with values along with equidistant time intervals $\mathrm{n} \tau$ with $\mathrm{n}=0,1, \ldots, \mathrm{N}-1$. The FDM aims to represent $c_{n}$ as a sum of damped sinusoids, as shown in (2), where $\omega_{k}=2 \pi f_{k}-j \gamma_{k}$ are the complex frequencies of the signal, including the damping factor, and $d_{k}$ are the corresponding amplitudes. To solve (1), the FDM associates a correlation function described by the Hamiltonian operator $\hat{\Omega}$, which has complex eigenvalues $\left\{\omega_{k}\right\}$, thus $c_{n}$ can be further transformed into (3):

$$
\begin{aligned}
& c_{n}=\sum_{k=1}^{K} d_{k} e^{-j n \tau \omega_{k}} \\
& c_{n}=\left(\Phi_{0} \mid e^{-j n \tau \Omega} \Phi_{0}\right)
\end{aligned}
$$

The problem can be simplified to the diagonalization of the Hamiltonian operator $\widehat{\Omega}$ or, as discussed in [28], to the evolution operator $\widehat{U}=e^{-j \tau \Omega}$. Briefly, a symmetric internal product operator defined by $(\mathrm{a} \mid \mathrm{b})=(\mathrm{b} \mid \mathrm{a})$ without the conjugate complex is used, where $\Phi_{0}$ is the initial state. Assuming that an orthonormal eigenvector set $\gamma_{k}$ is used to perform diagonalization of the evolution operator as shown in (4):

$$
\left.\widehat{U}=\sum_{k} u_{k} \mid Y_{k}\right)\left(Y_{k}\left|=\sum_{k} e^{-j \omega_{k} \tau}\right| Y_{k}\right)\left(Y_{k} \mid\right.
$$

and substituting (4) in (3), yields (5):

$$
d_{k}=\left(\Phi_{0} \mid Y_{k}\right)\left(Y_{k} \mid \psi_{0}\right)=\left(Y_{k} \mid \Phi_{0}\right)^{2}
$$

The resulting eigenvalues determine the position and width of the harmonics while the eigenvectors define their amplitudes and phases. Assuming a set created from the Krylov vectors, generated by the evolution operator $\Phi_{n}=U^{n} \Phi_{0}=e^{-j n \tau \widehat{\Omega}} \Phi_{0}$ and according to (5), it yields:

$$
\left(\Phi_{n} \mid \widehat{U} \Phi_{m}\right)=\left(\Phi_{n} \mid \Phi_{m+1}\right)=c_{m+n+1}
$$

as the set in non-orthonormal, the overlapping matrix can be calculated according to (7):

$$
\left(\Phi_{n} \mid \Phi_{m}\right)=\left(\widehat{U}^{n} \Phi_{0} \mid U^{m} \Phi_{0}\right)=\left(\Phi_{0} \mid \widehat{U}^{m+n} \Phi_{0}\right)=c_{m+n+1}
$$

In (7) is strictly related to the values of the measured signal. Notation $\mathrm{U}^{0}$ can then be used, being this the overlapping matrix representation of dimensions $M+1 \times M+1$. Similarly, $U^{1}$ can be used for $\widehat{U}$. To reformulate (2), it is then necessary to solve the generalized eigenvalues problem as shown in (8):

$$
U^{1} B_{k}=u_{k} U^{0} B_{k}
$$

where $u_{k}=e^{-j n \omega_{k} \tau}$ contains the lines of the spectrum and its corresponding widths. Eigenvectors $B_{k}$ contain both amplitudes and phases.

\subsubsection{FDM implementation for the e-nose}

With the aim of analyzing the sensors' experimental data, the FDM was implemented. The sensors' readings were used as inputs $c_{n}$, and the FDM was used to estimate their spectra. The following steps were performed by the algorithm:

- Taking into account the Nyquist criterion, the frequency interval $\left[f_{\min } f_{\max }\right]$ in which the spectral analysis of signal $c_{n}$ will be performed is selected; $c_{n}$ is sampled at $f_{s}=\frac{1}{\tau}$

- An angular frequency equidistant axis with values $2 \pi \mathrm{f}_{\min }<\varphi \mathrm{j}<2 \pi \mathrm{f}_{\max }, \mathrm{j}=0,1,2, \ldots, \mathrm{K}_{\text {win }}$ is created. Value $\mathrm{K}_{\text {win }}$ is chosen as $K_{\text {win }}=\frac{N\left(f_{\max }-f_{\min }\right)}{2 \tau}$ as suggested in the literature

- Three symmetric complex matrices $U^{(p)}$ of dimensions $K_{\text {win }} \times K_{\text {win }}$, with $\mathrm{p}=0,1,2$ are determined. To calculate the elements that do not belong to the diagonal, (9) can be used, where $f_{p}$ and $g_{p}$ are the Fourier transforms of the first and second part of signal $c_{n}$ [31], detailed in (10). 


$$
\begin{aligned}
& U^{(p)}\left(\varphi, \varphi^{\prime}\right)=\frac{e^{j \varphi} f_{p}\left(\varphi^{\prime}\right)-e^{j \varphi^{\prime}} f_{p}(\varphi)+e^{j M \varphi^{\prime}} g_{p}(\varphi)+e^{j M \varphi} g_{p}\left(\varphi^{\prime}\right)}{e^{-j \varphi}-e^{-j \varphi^{\prime}}} \\
& f_{p}(\varphi)=\sum_{n=0}^{M} e^{j n \varphi} c_{n+p} \\
& g_{p}(\varphi)=\sum_{n=M+1}^{2 M} e^{j(n-m-1) \varphi} c_{n+p}
\end{aligned}
$$

In (11) is used to calculate the elements located in the diagonal:

$$
U^{(p)}\left(\varphi, \varphi^{\prime}\right)=\sum_{n=0}^{2 M}(M+1-|m-n|) e^{j n \varphi}
$$

- Solve the generalized eigenvalues problem with (8), where the eigenvalues and eigenvectors are calculated using the QZ factorization algorithm [32].

- Select the complex amplitudes $d_{k}$ using (12):

$$
d_{k}^{1 / 2}=\sum_{j=1}^{K_{\text {win }}} \boldsymbol{B}_{j k} \sum_{n=0}^{M} c_{n} e^{j n \varphi_{j}}
$$

- Use values $\omega_{k}$ and $d_{k}$ to estimate the spectrum with (13). Figure 8 shows a flowchart summarizing the FDM algorithm implementation. The following section will verify its performance and suitability for enose data processing.

$$
C(F)=-\sum_{k} \operatorname{Im}\left\{\frac{d_{k}}{2 \pi F-\omega_{k}}\right\}
$$

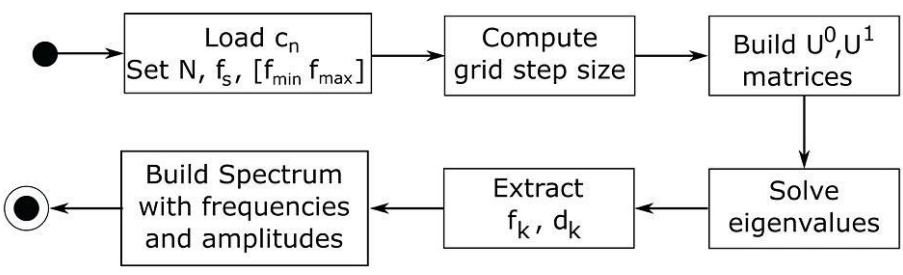

Figure 8. FDM implementation algorithm for the e-nose

\section{RESULTS AND DISCUSSION}

\subsection{E-nose data processing with FMD}

A series of experiments were designed using common gases to test the performance of the device, obtaining some preliminary results that are presented in this Section. The environmental conditions registered during the experiments were a temperature of $23{ }^{\circ} \mathrm{C} \pm 2{ }^{\circ} \mathrm{C}$ and relative humidity $(\mathrm{RH})$ of $30 \%$. For each experiment, the e-nose was subjected to a gas sample, and two features were obtained: i) the sensors response in the time domain consisted of a series of 180 samples for each of the six sensors; ii) the frequency spectrum for each sensor response was calculated using the FDM described in section 2.2. The spectrum of a sensor is given by (14):

$$
Y_{n}(f)=\sum_{k} a_{k, n} \delta\left(f-f_{k, n}\right)
$$

where $f_{k}$ and $a_{k}$ are the frequencies and amplitudes belonging to the $\mathrm{k}^{\text {th }}$-harmonic found in the response of the $\mathrm{n}^{\text {th }}$-sensor, respectively. The system response to clean air was first verified. As expected, the sensor set 
showed constant low magnitude output values. The FDM-based spectrum revealed low amplitude peaks and frequencies with inexistent harmonics. It can be therefore concluded that the sensors' response to clean air is negligible. Figure 9 shows the e-nose response to acetone $\left(\mathrm{C}_{3} \mathrm{H}_{6} \mathrm{O}\right)$. Note in Figure 9(a) how the sensors respond to the stimulus represented by the dotted line, especially sensors S4 and S2 followed by S6, this last indicating the air quality. In addition, a spectrum containing harmonics of relevant magnitude in a narrow frequency range can be observed as shown in Figure 9(b). Table 2 summarizes the frequency (Sn_F) and amplitude (Sn_A) values found by the FDM algorithm.

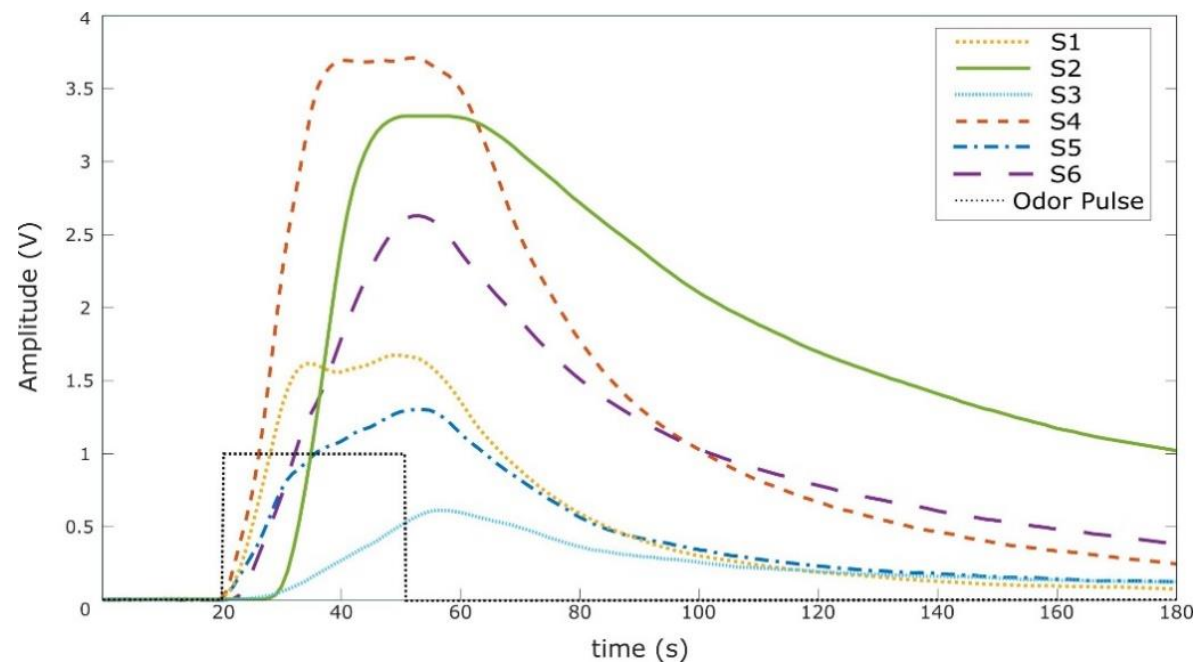

(a)
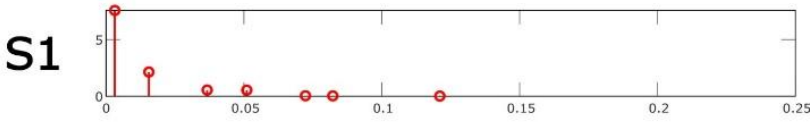

$\mathrm{S} 2$

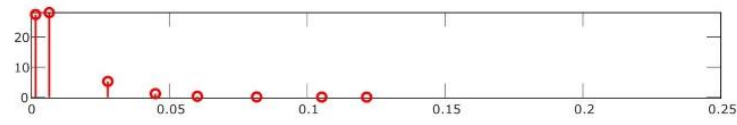

S3

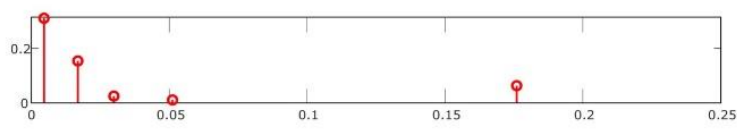

S4

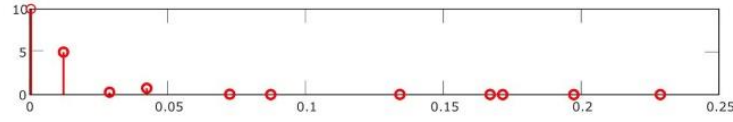

S5

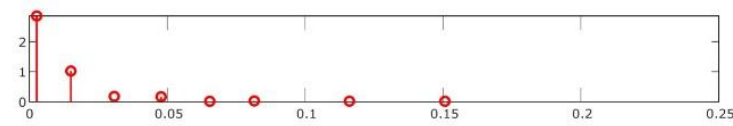

S6

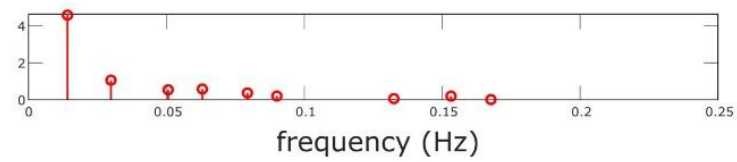

(b)

Figure 9. The e-nose response to acetone on (a) time response and (b) FDM-based spectrum

Figures 10(a) and (b) show the time response and FDM-based spectrum to ethanol at $71.5 \%$, respectively. As shown in Figure 10(a), sensors S2 and S4 exhibit the most significant responses; while S2 shows the highest amplitude value, S4 shows the fastest rise time. Table 3 lists the harmonic values found by the FDM algorithm. Note the correspondence with Figure 10(b), values obtained from S2 and S4 outstand from the rest of the sensors. 
Table 2. Acetone: amplitude (Sn_A) and frequency (Sn_F) values got for each sensor reading using FDM

\begin{tabular}{cccccccccccc}
\hline S1_A & S1_F & S2_A & S2_F & S3_A & S3_F & S4_A & S4_F & S5_A & S5_F & S6_A & S6_F \\
\hline 0.039 & 0.464 & 27.466 & 0.001 & 0.063 & 0.176 & 0.012 & 0.433 & 2.859 & 0.003 & 4.580 & 0.014 \\
7.571 & 0.003 & 28.083 & 0.006 & 0.311 & 0.005 & 0.016 & 0.410 & 1.024 & 0.015 & 1.061 & 0.030 \\
2.132 & 0.016 & 5.256 & 0.028 & 0.154 & 0.017 & 10.011 & 0 & 0.178 & 0.031 & 0.542 & 0.051 \\
0.545 & 0.037 & 1.195 & 0.045 & 0.025 & 0.030 & 4.978 & 0.012 & 0.166 & 0.048 & 0.584 & 0.063 \\
0.539 & 0.051 & 0.322 & 0.060 & 0.011 & 0.051 & 0.295 & 0.029 & 0.015 & 0.065 & 0.373 & 0.079 \\
0.041 & 0.072 & 0.110 & 0.082 & & & 0.761 & 0.042 & 0.027 & 0.082 & 0.206 & 0.090 \\
0.031 & 0.082 & 0.071 & 0.105 & & & 0.058 & 0.073 & 0.019 & 0.116 & 0.060 & 0.132 \\
0.011 & 0.121 & 0.041 & 0.122 & & & 0.020 & 0.087 & 0.016 & 0.151 & 0.201 & 0.153 \\
& & & & & & 0.022 & 0.229 & & & 0.015 & 0.168 \\
& & & & & 0.015 & 0.197 & & & & \\
& & & & & 0.034 & 0.134 & & & & \\
& & & & & 0.014 & 0.172 & & & & \\
\hline
\end{tabular}

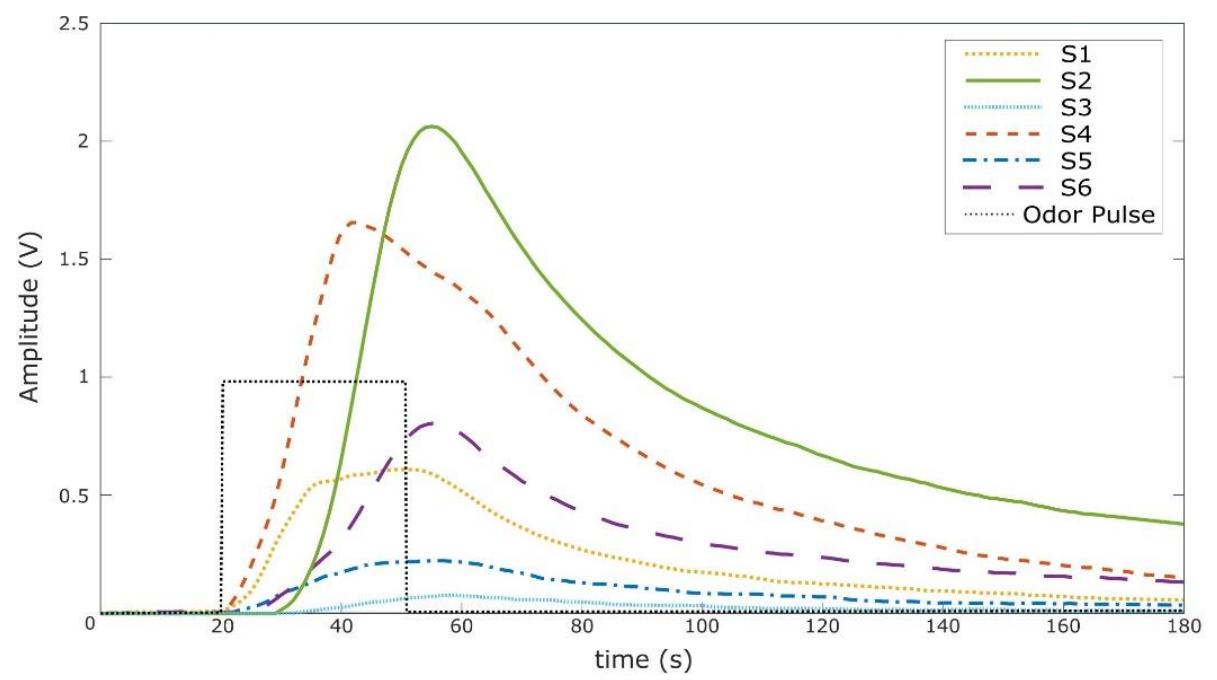

(a)
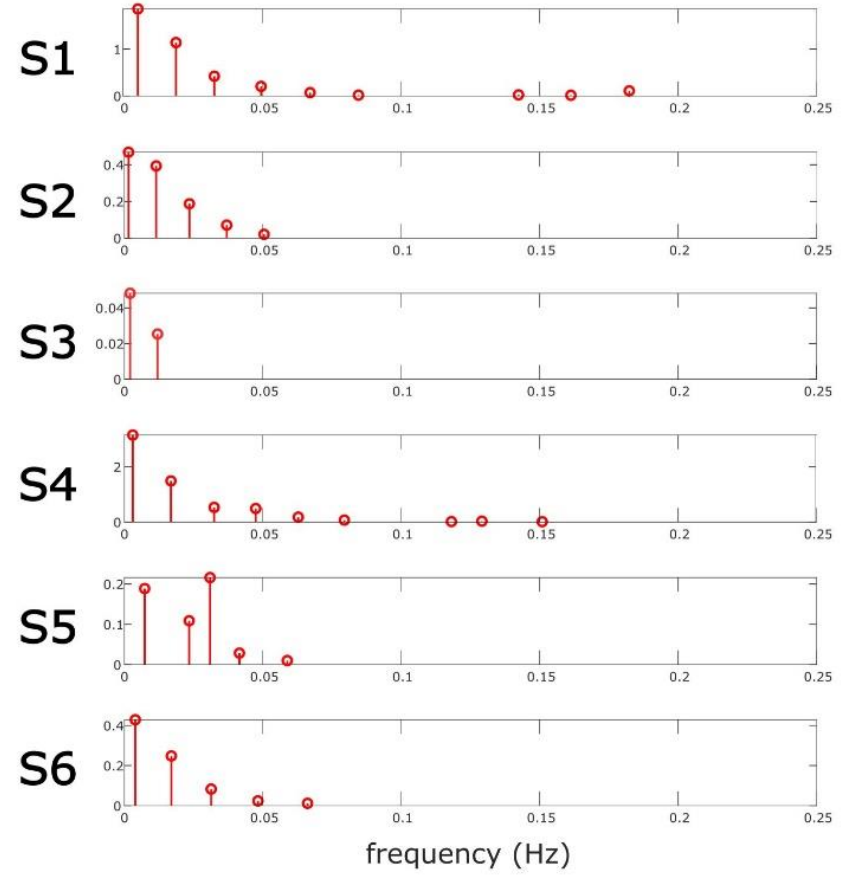

(b)

Figure 10. The e-nose response to ethanol on (a) time response and (b) FDM-based spectrum 
Table 3. Ethanol: amplitude (Sn_A) and frequency $\left(\mathrm{Sn} \_\right.$F) values got for each sensor reading using FDM

\begin{tabular}{llllllllllll}
\hline S1_A & S1_F & S2_A & S2_F & S3_A & S3_F & S4_A & S4_F & S5_A & S5_F & S6_A & S6_F \\
\hline 0.012 & 0.423 & 0.468 & 0.002 & 0.048 & 0.002 & 3.146 & 0.003 & 0.189 & 0.007 & 0.428 & 0.004 \\
1.852 & 0.005 & 0.393 & 0.011 & 0.025 & 0.012 & 1.487 & 0.017 & 0.109 & 0.023 & 0.248 & 0.017 \\
1.135 & 0.019 & 0.187 & 0.023 & & & 0.531 & 0.032 & 0.216 & 0.031 & 0.083 & 0.031 \\
0.421 & 0.032 & 0.071 & 0.037 & & & 0.489 & 0.047 & 0.029 & 0.042 & 0.024 & 0.048 \\
0.203 & 0.049 & 0.021 & 0.050 & & & 0.178 & 0.063 & 0.010 & 0.059 & 0.012 & 0.066 \\
0.071 & 0.067 & & & & & 0.071 & 0.079 & & & & \\
0.017 & 0.085 & & & & & 0.018 & 0.118 & & & & \\
0.108 & 0.182 & & & & & 0.032 & 0.129 & & & & \\
0.023 & 0.142 & & & & & 0.013 & 0.151 & & & & \\
0.015 & 0.161 & & & & & & & & & & \\
\hline
\end{tabular}

Finally, Figures 11(a) and (b) show the time response and FDM-based spectrum to gas butane $\left(\mathrm{C}_{4} \mathrm{H}_{10}\right)$, respectively. Note in Figure 11(a) that $\mathrm{S} 4$ exhibits the highest amplitude value. Table 4 shows the harmonics found by the FDM analysis. Note the correspondence with Figure 11(b), values obtained from S4 outstand from the rest of the sensors.

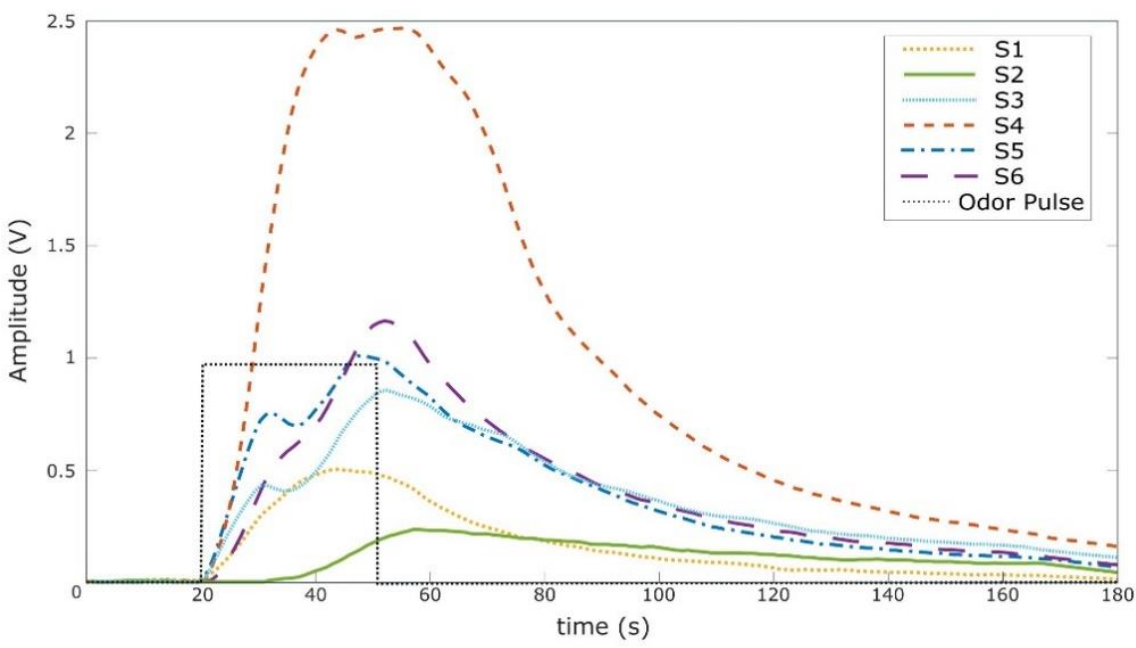

(a)
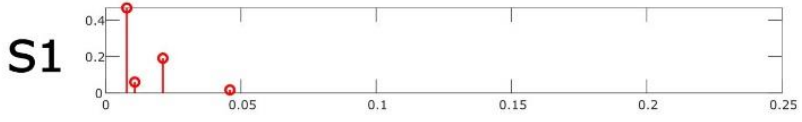

S2

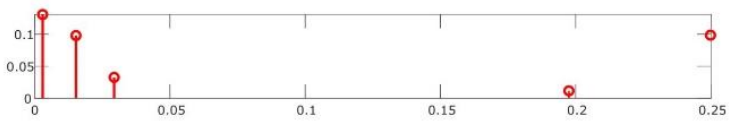

S3

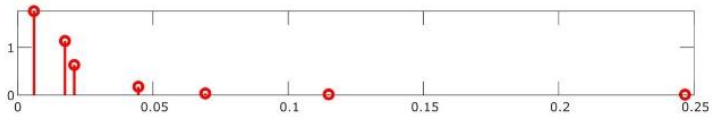

S4

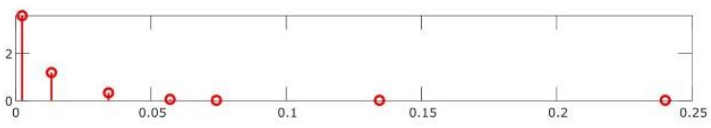

S5

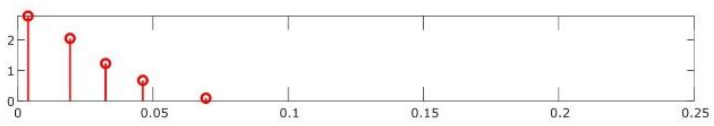

S6

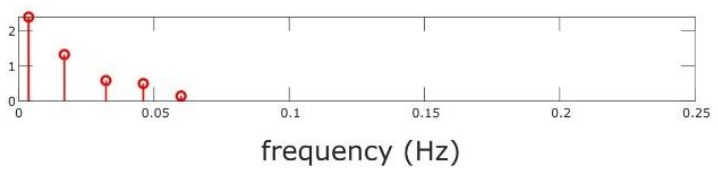

(b)

Figure 11. The e-nose response to gas butane on (a) time response and (b) FDM-based spectrum 
Table 4. Gas butane: amplitude (Sn_A) and frequency (Sn_F) values got for each sensor reading using FDM

\begin{tabular}{llllllllllll}
\hline S1_A & S1_F & S2_A & S2_F & S3_A & S3_F & S4_A & S4_F & S5_A & S5_F & S6_A & S6_F \\
\hline 0.060 & 0.011 & 0.131 & 0.003 & 1.760 & 0.006 & 3.603 & 0.002 & 2.782 & 0.004 & 2.388 & 0.004 \\
0.467 & 0.008 & 0.098 & 0.015 & 1.136 & 0.017 & 1.191 & 0.013 & 2.049 & 0.019 & 1.322 & 0.017 \\
0.191 & 0.021 & 0.033 & 0.029 & 0.632 & 0.021 & 0.333 & 0.034 & 1.230 & 0.033 & 0.581 & 0.032 \\
0.017 & 0.046 & 0.012 & 0.197 & 0.175 & 0.045 & 0.056 & 0.057 & 0.677 & 0.046 & 0.496 & 0.046 \\
& & & & 0.036 & 0.069 & 0.014 & 0.074 & 0.095 & 0.070 & 0.140 & 0.060 \\
& & & & 0.015 & 0.115 & 0.016 & 0.240 & & & & \\
\hline
\end{tabular}

\subsection{Comparison with Fourier analysis}

The discrete Fourier transform (DFT) is the most commonly used method to solve the harmonic inversion problem (HIP) using the reliable and efficient fast Fourier transform (FFT) algorithm. However, the FFT is sensitive to time-frequency uncertainties which limit the resolution of the resulting spectrum. As the FFT spectrum resolution depends on the number of processed samples, the use of excessively large data sets is often necessary. In contrast, the FDM is a parametric method that, upon the use of linear algebra, extracts the parameters relevant for the construction of the signal spectrum. The FDM outperforms the FFT as it requires a lower amount of data to build the spectrum and does not restrain the spectrum resolution with uncertainties.

Figure 12 compares the FDM and FFT spectra for the acetone sample. The comparison is limited to the signals of sensors S2 and S4, which show the clearest response to acetone. Note that the FDM produces more accurate high-resolution spectra with well-defined peaks. Harmonics are well separated among them, clearly showing the signal frequencies in the spectrum. In contrast, the FFT produces wide peaks that tend to merge between them; this low-resolution effect might lose or miss information on the spectrum harmonics. Note that the FDM-based spectrum exhibits a higher number of harmonics represented by well-defined peaks. It can be therefore concluded that the FDM-based spectrum is a clearer and more accurate representation for the e-nose signal processing.

Current work focuses on using these spectra to perform gas classification. Approaches such as random forest [33] and convolutional neural networks (CNN) [34] are currently being considered. Future work will evaluate the possibility of optimizing the number of sensors while keeping satisfactory results and migrating the electronic unit to an field-programmable gate array (FPGA) system on chip (SoC) architecture [35]. Applications in wireless sensor networks (WSN) for domotics/inmotics [36] and automated farming [37] are foreseen.

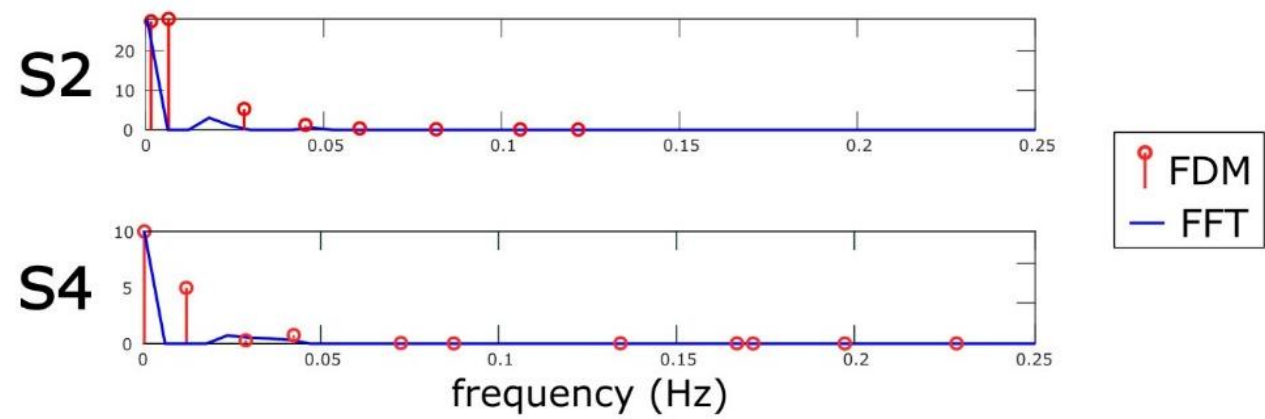

Figure 12. Performance comparison between FDM and FFT (180 samples)

\section{CONCLUSION}

This research work has described a self-developed e-nose device implemented with low-cost components. It is devoted to detect potentially harmful volatile compounds in the environment. The prototype involves an array of six metal oxide semiconductor (MOS) sensors, an electronic module for data acquisition, and a computer-based information system for signal analysis and visualization. MOS sensors offer small size, low power consumption, fast response, and recovery times. Their response can be divided in three timeregions: the reference value (baseline), the rising time, and the resetting time.

The hardware design and testing stages are now complete ensuring that the requirements have been fulfilled and that the prototype is functional. The filter diagonalization method (FDM) was implemented to calculate the harmonics involved in the acquired signals. The algorithm showed high precision using a low 
number of samples. To our knowledge, FDM has not been previously explored for e-nose data processing and has the potential to become a valuable tool for this application.

\section{REFERENCES}

[1] C. G. Viejo, S. Fuentes, A. Godbole, B. Widdicombe, and R. R. Unnithan, "Development of a low-cost e-nose to assess aroma profiles: An artificial intelligence application to assess beer quality," Sensors and Actuators, B: Chemical, vol. 308, Apr. 2020, Art. no. 127688 , doi: $10.1016 /$ j.snb.2020.127688

[2] B. Radi, D. N. Rohmah, E. Wahyudi, M. D. Adhityamurti, and J. P. L. Yuroto Putro, "Implementation of an electronic nose for classification of synthetic flavors," Bulletin of Electrical Engineering and Informatics (BEEI), vol. 10, no. 3, pp. 1283-1290, Jun. 2021, doi: 10.11591/eei.v10i3.3018.

[3] K. Xu, J. Wang, Z. Wei, F. Deng, Y. Wang, and S. Cheng, “An optimization of the MOS electronic nose sensor array for the detection of Chinese pecan quality," Journal of Food Engineering, vol. 203, pp. 25-31, Jun. 2017, doi: 10.1016/j.jfoodeng.2017.01.023.

[4] K. Timsorn and C. Wongchoosuk, "Inkjet printing of room-temperature gas sensors for identification of formalin contamination in squids," Journal of Materials Science: Materials in Electronics, vol. 30, no. 5, pp. 4782-4791, Jan. 2019, doi: 10.1007/s10854019-00772-9.

[5] A. Gliszczyńska-Świgło and J. Chmielewski, "Electronic nose as a tool for monitoring the authenticity of food. a review," Food Analytical Methods, vol. 10, no. 6, pp. 1800-1816, Dec. 2017, doi: 10.1007/s12161-016-0739-4.

[6] S. A. Laga and R. Sarno, "Temperature effect of electronic nose sampling for classifying mixture of beef and pork," Indonesian Journal of Electrical Engineering and Computer Science (IJEECS), vol. 19, no. 3, pp. 1626-1634, Sep. 2020, doi: 10.11591/ijeecs.v19.i3.pp1626-1634

[7] M. Wang et al., "Real-time assessment of food freshness in refrigerators based on a miniaturized electronic nose," Analytical Methods, vol. 10, no. 39, pp. 4741-4749, 2018, doi: 10.1039/c8ay01242c.

[8] C. Sánchez, J. P. Santos, and J. Lozano, "Use of electronic noses for diagnosis of digestive and respiratory diseases through the breath," Biosensors, vol. 9, no. 1, p. 35, Feb. 2019, doi: 10.3390/bios9010035.

[9] S. Siyang, C. Wongchoosuk, and T. Kerdcharoen, "Diabetes diagnosis by direct measurement from urine odor using electronic nose," in The 5th 2012 Biomedical Engineering International Conference, Dec. 2012, pp. 1-4, doi: 10.1109/BMEiCon.2012.6465441.

[10] M. Taylor, "Airbus wants its bomb-detecting 'E-nose' to sense COVID-19." laboratoryequipment.com. https://www.laboratoryequipment.com/563979-Airbus-Wants-its-Bomb-detecting-E-nose-to-Sense-Viral-Diseases-Too/ (accessed Nov. 16, 2021).

[11] P. Jia, F. Tian, Q. He, S. Fan, J. Liu, and S. X. Yang, "Feature extraction of wound infection data for electronic nose based on a novel weighted KPCA," Sensors and Actuators, B: Chemical, vol. 201, pp. 555-566, Oct. 2014, doi: 10.1016/j.snb.2014.05.025.

[12] N. Peled et al., "Detection of volatile organic compounds in cattle naturally infected with Mycobacterium bovis," Sensors and Actuators, B: Chemical, vol. 171-172, pp. 588-594, Aug. 2012, doi: 10.1016/j.snb.2012.05.038.

[13] M. Baietto, A. D. Wilson, D. Bassi, and F. Ferrini, "Evaluation of three electronic noses for detecting incipient wood decay," Sensors, vol. 10, no. 2, pp. 1062-1092, Jan. 2010, doi: 10.3390/s100201062.

[14] A. D. Wilson, "Identification of insecticide residues with a conducting-polymer electronic nose," Chemical Sensors, vol. 4, no. 3, pp. 1-10, 2014.

[15] L. Capelli, S. Sironi, and R. Del Rosso, "Electronic noses for environmental monitoring applications," Sensors (Switzerland), vol. 14, no. 11, pp. 19979-20007, Oct. 2014, doi: 10.3390/s141119979.

[16] C. Wongchoosuk, C. Khunarak, M. Lutz, and T. Kerdcharoen, "WiFi electronic nose for indoor air monitoring," in 20129 th International Conference on Electrical Engineering/Electronics, Computer, Telecommunications and Information Technology, May 2012, pp. 1-4, doi: 10.1109/ECTICon.2012.6254166.

[17] S. O. Mishra and S. H. Saeed, "Optimization of electronic sensors for detecting pollution due to organic gases using PARAFAC," International Journal of Electrical and Computer Engineering (IJECE), vol. 9, no. 5, pp. 3441-3449, Oct. 2019, doi: 10.11591/ijece.v9i5.pp3441-3449.

[18] R. E. Baby, M. Cabezas, and E. N. W. De Reca, "Electronic nose: A useful tool for monitoring environmental contamination," Sensors and Actuators, B: Chemical, vol. 69, no. 3, pp. 214-218, Oct. 2000, doi: 10.1016/S0925-4005(00)00491-3.

[19] I. Iswanto, A. Ma'arif, B. Kebenaran, and P. Megantoro, "Design of gas concentration measurement and monitoring system for biogas power plant," Indonesian Journal of Electrical Engineering and Computer Science (IJEECS), vol. 22, no. 2, pp. 726-732, May 2021, doi: 10.11591/ijeecs.v22.i2.pp726-732.

[20] M. K. Ibrahim, N. M. Hussien, and S. N. Alsaad, "Smart system for monitoring ammonium nitrate storage warehouse," Indonesian Journal of Electrical Engineering and Computer Science (IJEECS), vol. 23, no. 1, pp. 583-589, Jul. 2021, doi: 10.11591/ijeecs.v23.i1.pp583-589.

[21] L. Wang, Y. H. Gui, S. P. Zhang, and C. S. Xie, "Research on explosives detection by electronic nose," Chinese Journal of Sensors and Actuators, vol. 20, no. 1, pp. 42-45, 2007

[22] R. C. Young, W. J. Buttner, B. R. Linnell, and R. Ramesham, "Electronic nose for space program applications," Sensors and Actuators, B: Chemical, vol. 93, no. 1-3, pp. 7-16, Aug. 2003, doi: 10.1016/S0925-4005(03)00338-1.

[23] B. Gunawan, S. Alfarisi, G. Satrio, A. Sudarmaji, M. Malvin, and K. Krisyarangga, "MOS gas sensor of meat freshness analysis on E-nose," TELKOMNIKA (Telecommunication Computing Electronics and Control), vol. 17, no. 2, pp. 771-780, Apr. 2019, doi: 10.12928/telkomnika.v17i2.11787.

[24] D. R. Wijaya, R. Sarno, and E. Zulaika, "Gas concentration analysis of resistive gas sensor array," in 2016 International Symposium on Electronics and Smart Devices, ISESD 2016, Nov. 2017, pp. 337-342, doi: 10.1109/ISESD.2016.7886744.

[25] M. Gancarz et al., "Application of electronic nose with MOS sensors to prediction of rapeseed quality," Measurement: Journal of the International Measurement Confederation, vol. 103, pp. 227-234, Jun. 2017, doi: 10.1016/j.measurement.2017.02.042.

[26] R. Macias-Quijas, R. Velazquez, N. I. Giannoccaro, and A. Lay-Ekuakille, "A novel electronic nose instrument for the detection of volatile hazardous compounds: preliminary results," in 2021 7th International Conference on Control, Instrumentation and Automation (ICCIA), Feb. 2021, pp. 1-5, doi: 10.1109/ICCIA52082.2021.9403595

[27] M. R. Wall and D. Neuhauser, "Extraction, through filter-diagonalization, of general quantum eigenvalues or classical normal mode frequencies from a small number of residues or a short-time segment of a signal. I. Theory and application to a quantumdynamics model," The Journal of Chemical Physics, vol. 102, no. 20, pp. 8011-8022, May 1995, doi: 10.1063/1.468999. 
[28] V. A. Mandelshtam, "FDM: The filter diagonalization method for data processing in NMR experiments," Progress in Nuclear Magnetic Resonance Spectroscopy, vol. 38, no. 2, pp. 159-196, Mar. 2001, doi: 10.1016/S0079-6565(00)00032-7.

[29] A. Lay-Ekuakille, C. Pariset, and A. Trotta, "Leak detection of complex pipelines based on the filter diagonalization method: Robust technique for eigenvalue assessment," Measurement Science and Technology, vol. 21, no. 11, Sep. 2010, Art. no. 115403, doi: 10.1088/0957-0233/21/11/115403

[30] A. Lay-Ekuakille, G. Vendramin, and A. Trotta, "Robust spectral leak detection of complex pipelines using filter diagonalization method," IEEE Sensors Journal, vol. 9, no. 11, pp. 1605-1614, Nov. 2009, doi: 10.1109/JSEN.2009.2027410

[31] V. A. Mandelshtam and H. S. Taylor, "Harmonic inversion of time signals and its applications," Journal of Chemical Physics, vol. 107, no. 17, pp. 6756-6769, Nov. 1997, doi: 10.1063/1.475324.

[32] D. J. Evans and W. S. Yousif, "The QZ algorithm for the calculation of the eigenvalues of a real matrix," Parallel Algorithms and Applications, vol. 4, no. 3-4, pp. 183-192, Jan. 1994, doi: 10.1080/10637199408915463.

[33] V. M. Álvarez-Pato, C. N. Sánchez, J. Domínguez-Soberanes, D. E. Méndoza-Pérez, and R. Velázquez, "A multisensor data fusion approach for predicting consumer acceptance of food products," Foods, vol. 9, no. 6, Jun. 2020, Art. no. 774, doi: 10.3390/foods9060774.

[34] B. Calabrese, R. Velázquez, C. Del-Valle-Soto, R. de Fazio, N. I. Giannoccaro, and P. Visconti, "Solar-powered deep learningbased recognition system of daily used objects and human faces for assistance of the visually impaired," Energies, vol. 13, no. 22, Nov. 2020, Art. no. 6104,doi: 10.3390/en13226104.

[35] P. Visconti, R. Velazquez, S. Capoccia, and R. De Fazio, "High-performance AES-128 algorithm implementation by FPGA-based SoC for 5G communications," International Journal of Electrical and Computer Engineering (IJECE), vol. 11, no. 5, pp. 42214232, Oct. 2021, doi: 10.11591/ijece.v11i5.pp4221-4232.

[36] C. Del-Valle-Soto, L. J. Valdivia, R. Velázquez, L. Rizo-Dominguez, and J. C. López-Pimentel, "Smart campus: An experimental performance comparison of collaborative and cooperative schemes for wireless sensor network," Energies, vol. 12, no. 16, Aug. 2019, Art. no. 3135, doi: 10.3390/en12163135.

[37] P. Visconti, N. I. Giannoccaro, R. de Fazio, S. Strazzella, and D. Cafagna, "IoT-oriented software platform applied to sensorsbased farming facility with smartphone farmer app," Bulletin of Electrical Engineering and Informatics (BEEI), vol. 9, no. 3, pp. 1095-1105, Jun. 2020, doi: 10.11591/eei.v9i3.2177. 